\title{
Cytolethal distending toxin B as a cell-killing component of tumor-targeted anthrax toxin fusion proteins
}

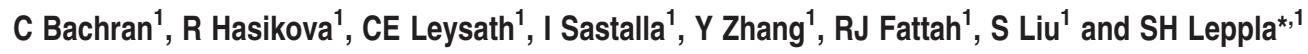

Cytolethal distending toxin (Cdt) is produced by Gram-negative bacteria of several species. It is composed of three subunits, CdtA, CdtB, and CdtC, with CdtB being the catalytic subunit. We fused CdtB from Haemophilus ducreyi to the N-terminal 255 amino acids of Bacillus anthracis toxin lethal factor (LFn) to design a novel, potentially potent antitumor drug. As a result of this fusion, CdtB was transported into the cytosol of targeted cells via the efficient delivery mechanism of anthrax toxin. The fusion protein efficiently killed various human tumor cell lines by first inducing a complete cell cycle arrest in the G2/M phase, followed by induction of apoptosis. The fusion protein showed very low toxicity in mouse experiments and impressive antitumor effects in a Lewis Lung carcinoma model, with a $90 \%$ cure rate. This study demonstrates that efficient drug delivery by a modified anthrax toxin system combined with the enzymatic activity of CdtB has great potential as anticancer treatment and should be considered for the development of novel anticancer drugs.

Cell Death and Disease (2014) 5, e1003; doi:10.1038/cddis.2013.540; published online 16 January 2014

Subject Category: Cancer

The use of anthrax toxin proteins to target tumor cells has been studied extensively in the past decade. Studies have shown that lethal factor (LF) of anthrax toxin is a potent antiangiogenic drug that dramatically slows tumor growth, while not directly targeting tumor cells. ${ }^{1}$

Anthrax toxin is produced by Bacillus anthracis, a Grampositive, rod-shaped bacterium that is the causative agent of anthrax. The toxin has three components: the cell-binding moiety protective antigen (PA), LF, and edema factor (EF). Upon binding of PA to the cellular receptors (tumor endothelial marker 8 and capillary morphogenesis gene 2), it is cleaved and thereby activated by host furin and furin-like proteinases. Activated PA forms an oligomer (heptamer or octamer ${ }^{2}$ ), and allows for LF and EF binding. The complex is then endocytosed and upon acidification in endosomes, the catalytic subunits are translocated into the cytosol through a pore formed by the PA oligomer. LF is a metalloprotease that cleaves and inhibits the mitogen-activated protein kinase pathways. These pathways are responsible for recognizing a variety of external signals and inducing protective cellular responses, such as inflammation and apoptosis. EF, the second catalytic toxin component, is a calcium- and calmodulin-dependent adenylate cyclase and elevates intracellular cyclic AMP (cAMP) concentrations. cAMP functions as a second messenger and is responsible for several cellular responses.

Cytolethal distending toxin $(\mathrm{Cdt})$ is another tripartite bacterial protein toxin that is produced by several
Gram-negative bacteria, including the human pathogens Escherichia coli, Haemophilus ducreyi, Aggregatibacter actinomycetemcomitans, Campylobacter jejuni, and Shigella dysenteriae. Cdt is composed of the three subunits $\mathrm{CdtA}$, CdtB, and CdtC. The Cdt of $H$. ducreyi appears to be a virulence factor of the bacterium in a chancroid model of infection and was subsequently studied as a potential vaccine, with promising results. ${ }^{3,4}$

CdtA and CdtB were originally discovered by Lagergard et al., ${ }^{4}$ who documented the presence of the toxin in $\mathrm{H}$. ducreyi supernatant fluids and who demonstrated its toxic activity against several human cell lines. ${ }^{5} \mathrm{Cdt} A$ and $\mathrm{CdtC}$ are required for receptor binding and translocation of the enzymatically active $\mathrm{CdtB}$ component. The mammalian cell receptor for the $H$. ducreyiCdt was recently determined by a genetic screen to be TMEM181. ${ }^{6}$ The same approach identified sphingomyelin synthase 1 as a gene required for Cdt activity, probably due to the involvement of lipid rafts in receptor clustering. The detailed roles of $\mathrm{CdtA}$ and $\mathrm{CdtC}$ remain unclear.

CdtB has been suggested to be a metal-dependent DNase that causes degradation of nuclear DNA in host cells. ${ }^{7} \mathrm{CdtB}$ has high sequence homology to DNase I, and crystal structures confirmed the homology between the two enzymes. Treatment with Cdt induces G2/M phase cell cycle arrest and subsequently apoptosis in many mammalian cell types. $^{8}$ A recent study also suggested that CdtB exhibits phosphatase activity on the plasma membrane-associated

\footnotetext{
${ }^{1}$ Laboratory of Parasitic Diseases, National Institute of Allergy and Infectious Diseases, National Institutes of Health, Bethesda, MD, USA

*Corresponding author: SH Leppla, Laboratory of Parasitic Diseases, National Institute of Allergy and Infectious Diseases, National Institutes of Health, Bethesda, MD 20892, USA. Tel: +1 301594 2865; Fax: +1 301480 3633; E-mail: sleppla@ niaid.nih.gov

Keywords: immunotoxin; targeted toxin; anthrax; drug delivery; cytolethal distending toxin

Abbreviations: Cdt, cytolethal distending toxin; CdtB, Cdt subunit B; EF, edema factor; FP59AGG, fusion protein of LFn and Pseudomonas exotoxin A catalytic domain; LF, lethal factor; LFn, lethal factor N-terminal domain; LFnCdtB, fusion protein of LFn and CdtB; PA, wild-type protective antigen; PA-L1, PA with matrix metalloproteinase cleavage site; SI, survival index; $\mathrm{SI}_{50}, 50 \%$ survival index

Received 3.10.13; revised 27.11.13; accepted 2.12.13; Edited by A Stephanou
} 
signaling molecule phosphatidylinositol-3,4,5-triphosphate (PIP3). ${ }^{9}$ Shenker et al. ${ }^{9}$ identified a structural homology between CdtB and the tumor suppressor phosphatase and tensin homolog (PTEN) and demonstrated both in vitro and cellular phosphatase activity of CdtB.

In this study, we designed a fusion protein containing the $\mathrm{N}$-terminal 255 amino acids of LF (LFn, required for PA binding and translocation) and $\mathrm{CdtB}$ from $H$. ducreyi. We analyzed the cytotoxicity of the fusion protein fusion protein of LFn and CdtB (LFnCdtB) in combination with PA on a variety of tumor cells lines and performed mouse experiments to determine the toxicity and efficacy of the fusion protein in a murine tumor model.

\section{Results}

Design of LFnCdtB. LFnCdtB was successfully purified from a non-virulent, plasmid-cured, protease-deficient $B$. anthracis strain. ${ }^{10}$ The protein was secreted to the supernatant and purified in yields of at least $0.8 \mathrm{mg}$ per liter of culture (Supplementary Figure S1). The molecular mass of the protein was confirmed by electrospray ionization mass spectrometry (Supplementary Figure S2). A fusion protein, consisting of LFn and the catalytic domain of Pseudomonas exotoxin A (FP59AGG, similar to $F P 59^{11}$ but with the wildtype AGG N-terminus of LF) was used as a control for effective cell killing. FP59AGG efficiently prevents protein synthesis by enzymatically modifying elongation factor-2. We confirmed the catalytic activity of LFnCdtB in comparison with
CdtB in a DNA cleavage assay (Supplementary Text S1). The CdtB within LFnCdtB showed enzymatic activity in a similar manner as CdtB (Supplementary Figure S3).

Cytotoxicity of LFnCdtB. Cytotoxicity analyses used RAW264.7, CHO K1, HeLa, and HN6 cells. A 72-h exposure to $250 \mathrm{ng} / \mathrm{ml} \mathrm{PA}+\mathrm{LFnCdtB}$ in varying concentrations resulted in dose-dependent cytotoxicity with cytotoxicity in the order $\mathrm{CHO} \mathrm{K} 1>\mathrm{RAW}=\mathrm{HeLa}>\mathrm{HN} 6$ (Figures 1a-d). The $50 \%$ survival index $\left(\mathrm{SI}_{50}\right)$ values are shown in Table 1 . The observed $\mathrm{SI}_{50}$ values are in the range of $0.5 \mathrm{pM}(\mathrm{CHO} \mathrm{K1})$ to $142 \mathrm{pM}$ (HN6). The cells were also incubated with $250 \mathrm{ng} / \mathrm{ml}$ $\mathrm{PA}+\mathrm{CdtB}$, and no evidence of cytotoxicity was observed at concentrations up to $100 \mathrm{nM} \mathrm{CdtB}$. To compare the activity of LFnCdtB with prior established tumor-targeting fusion proteins $^{12}$ and as a prerequisite for subsequent experiments in an established tumor model murine LL3 cells were incubated with $250 \mathrm{ng} / \mathrm{ml}$ wild-type PA or $250 \mathrm{ng} / \mathrm{ml}$ matrix metalloproteinase-2-activated PA-L1 (PA-L1) in combination with LFnCdtB and FP59AGG at varying concentrations (Figure 1e). PA-L1 contains a mutated protease cleavage site to render the activation of PA dependent on the tumorassociated protease matrix metalloproteinase-2 instead of furin and furin-like proteases. ${ }^{12}$ Incubation with PA and PA-L1 resulted in similar cytotoxicities for both LFn fusion proteins $\left(\mathrm{SI}_{50}\right.$ values for $\mathrm{LFnCdtB}$ were $0.76 \mathrm{nM}$ and $0.33 \mathrm{nM}$, respectively, and $\mathrm{SI}_{50}$ values for FP59AGG were $0.026 \mathrm{nM}$ and $0.076 \mathrm{nM}$, respectively). FP59AGG was more cytotoxic, but the marginal differences between wild-type PA and
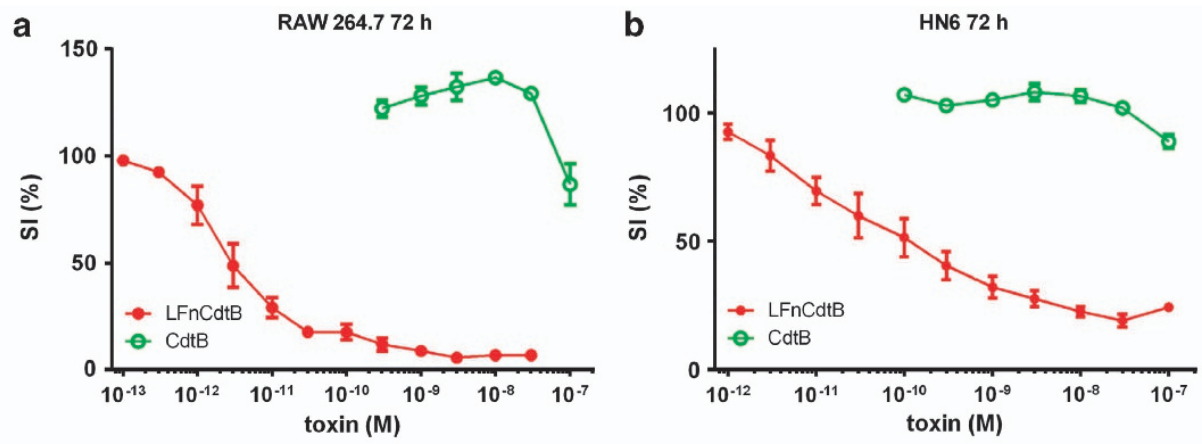

C

HeLa 72 h

d

CHO K1 $72 \mathrm{~h}$

e

LL3 $72 \mathrm{~h}$
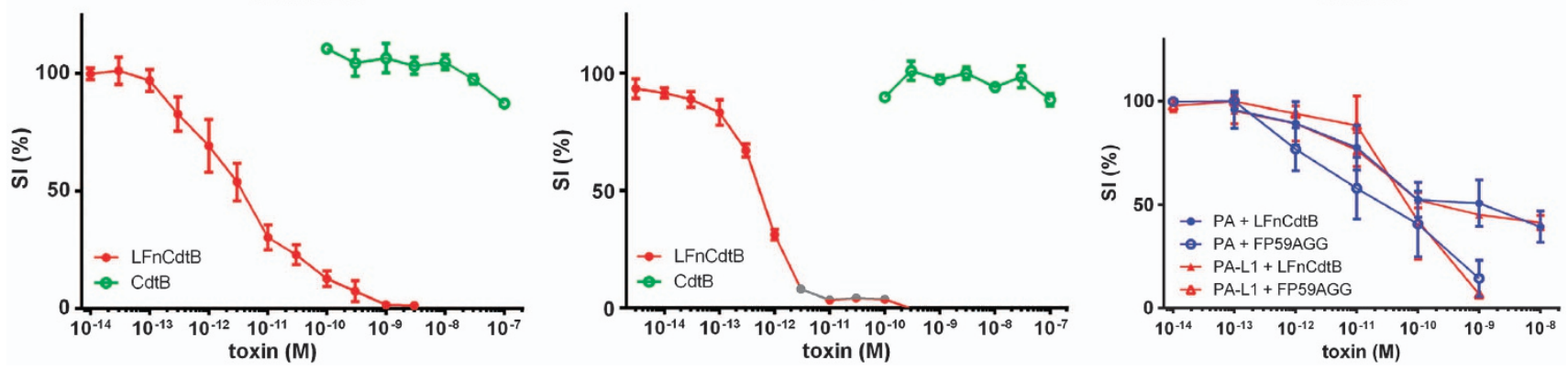

Figure 1 Cytotoxicity of fusion proteins on RAW264.7 cells (murine leukemic monocyte/macrophages), CHO K1 cells (Chinese Hamster Ovary cells), HeLa cells (human cervical carcinoma cell line), HN6 cells (human head and neck cancer cell line), and LL3 cells (murine Lewis Lung carcinoma cells). Cells (5000/well grown overnight) were exposed to different concentrations of fusion proteins for $72 \mathrm{~h}$. All samples contained a fixed concentration of $250 \mathrm{ng} / \mathrm{ml} \mathrm{PA}$ or tumor-specific PA-L1 (e) and varying concentrations of LFnCdtB or CdtB (a-d) or LFnCdtB or FP59AGG (e). Viable cells were quantitated in an assay employing 3-(4,5-Dimethylthiazol-2-yl)-2,5diphenyltetrazolium bromide (MTT). Relative survival was calculated as the percentage of living cells after treatment in relation to untreated cells. Error bars indicate S.E.M. of 2 (CdtB) and 3-6 (LFnCdtB and FP59AGG) independent experiments performed in triplicate 
PA-L1 for both fusion proteins indicate successful activation of PA-L1 on LL3 cells and demonstrated the suitability of PA-L1/LFnCdtB for in vivo experiments on LL3 tumors.

We also tested the LFnCdtB toxin on 14 human tumor cell lines of the $\mathrm{NCl}-60$ cell panel from the National Cancer Institute and on two murine tumor cell lines (B16/BL6, a murine melanoma cell line and T241, a murine fibrosarcoma cell line). A number of cell lines were highly sensitive to LFnCdtB, with $\mathrm{SI}_{50}$ values in the $\mathrm{pM}$ range, whereas a few lines were highly resistant (Table 1).

Cell cycle arrest. The effect of LFnCdtB on the arrest of cycle cycle progression was studied in $\mathrm{CHO} \mathrm{K} 1$ cells. Propidium iodide staining demonstrated that treatment with PA + LFnCdtB induces a potent cell cycle arrest in the G2/M phase (Figure 2). Staurosporine was used as a control for apoptosis induction without affecting the cell cycle. A strong increase in the population of cells in the G2/M phase was detected after $8 \mathrm{~h}$ of toxin exposure. Nearly all cells were arrested in G2/M after $14 \mathrm{~h}$, at which point apoptotic cells with very low $\mathrm{PI}$ intensity began to be detected. Cells treated with PA + FP59AGG showed no cell cycle arrest after 24 or $48 \mathrm{~h}$ but a high fraction of apoptotic cells was detected after $48 \mathrm{~h}$.

Table $1 \mathrm{SI}_{50}$ values for the cytotoxicity of PA + LFnCdtB or PA + FP59AGG $\mathrm{NCl}-60$ panel cell lines and murine cell lines

\begin{tabular}{|c|c|c|}
\hline \multirow[t]{2}{*}{ Cell line } & \multicolumn{2}{|c|}{$\mathrm{SI}_{50}(\mathrm{nM})$} \\
\hline & LFnCdtB & FP59AGG \\
\hline \multicolumn{3}{|l|}{ Melanoma } \\
\hline C32 & 0.00035 & 0.0022 \\
\hline Malme-3M & $\gg 10$ & 0.0027 \\
\hline SK-MEL-2 & $\gg 10$ & 0.010 \\
\hline SK-MEL-24 & 0.77 & 0.017 \\
\hline SK-MEL-28 & $>10$ & 0.0021 \\
\hline \multicolumn{3}{|l|}{ Colon } \\
\hline COLO 205 & 0.11 & 0.008 \\
\hline HCC2998 & 0.085 & 0.0037 \\
\hline SW620 & 0.31 & 0.024 \\
\hline \multicolumn{3}{|l|}{ Lung } \\
\hline A5̆49 & 1.6 & 0.002 \\
\hline $\mathrm{NCl}-\mathrm{H} 226$ & $>10$ & 0.00043 \\
\hline \multicolumn{3}{|l|}{ Breast } \\
\hline MDA-MB-231 & 0.018 & 0.0068 \\
\hline Hs $578 \mathrm{~T}$ & 0.18 & 0.037 \\
\hline \multicolumn{3}{|l|}{ Kidney } \\
\hline A-498 & 0.072 & 0.008 \\
\hline SN12C & 0.083 & 0.00038 \\
\hline HN6 (head and neck) & 0.14 & ND \\
\hline HeLa (cervical) & 0.0037 & ND \\
\hline \multicolumn{3}{|l|}{ Murine/Chinese hamster } \\
\hline CHO K1 (Chinese hamster) & 0.00048 & ND \\
\hline RAW264.7 & 0.0037 & ND \\
\hline T241 & 0.27 & 0.064 \\
\hline LL3 (PA) & 0.76 & 0.026 \\
\hline LL3 (PA-L1) & 0.33 & 0.073 \\
\hline B16/BL6 (PA) & 0.021 & 0.0005 \\
\hline B16/BL6 (PA-L1) & 0.038 & 0.054 \\
\hline
\end{tabular}

Abbreviations: NA, not determined; PA, protective antigen; $\mathrm{SI}_{50}, 50 \%$ survival index
Apoptosis induction. Apoptosis induction by LFnCdtB was analyzed by poly-ADP-ribose-polymerase (PARP) cleavage in HeLa cells and by terminal deoxynucleotidyl transferase dUTP nick end labeling (TUNEL) staining in $\mathrm{CHO} \mathrm{K} 1$ cells. The PARP cleavage assay showed apoptosis induction by the positive control staurosporine after $2 \mathrm{~h}$ exposure on the cells (Figure 3a). PA + LFnCdtB induced a strong apoptotic signal after 48 and $72 \mathrm{~h}$, whereas, as expected, $\mathrm{PA}+\mathrm{CdtB}$ failed to induce apoptosis in HeLa cells and only a low amount of cleaved PARP was detected after 48 and $72 \mathrm{~h}$, which could have resulted from cells dying in the crowded culture dish. Interestingly, the amount of detectable actin in the LFnCdtBtreated samples was very low, probably reflecting the effect of prolonged apoptosis and cell loss in these cultures.

The TUNEL assay showed slightly increased numbers of apoptotic $\mathrm{CHO}$ K1 cells after PA + LFnCdtB treatment for 14 and $24 \mathrm{~h}$ (Figure 3b). After the 48-h toxin exposure more than $90 \%$ of cells were positive for TUNEL staining. Additional cultures treated with PA + FP59AGG yielded a result similar to that for $P A+L F n C d t B$. The number of apoptotic cells after $24 \mathrm{~h}$ appeared to be higher as a consequence of PA + FP59AGG treatment. Staurosporine induced a very strong increase in apoptotic cells after $4 \mathrm{~h}$ ( $50 \%$ of cells positive for TUNEL staining) and a complete conversion of cells (>96\% TUNEL-positive) after $8 \mathrm{~h}$, whereas PA + CdtB failed to induce apoptosis.

Intracellular localization and nuclear action. To allow for the sensitive detection by streptavidin within cells, LFnCdtB was biotinylated and detected by western blotting in both cytosolic and nuclear fractions of $\mathrm{CHO}$ K1 cells after 1 and $4 \mathrm{~h}$ fusion protein exposure (Figure 4a). Detection of marker proteins for the nucleus (p84) and the cytosol (MEK2) established the purity of the fractions. The endogenous biotinylated carboxylase proteins ${ }^{13}$ were also detected by streptavidin in the nuclear fraction (weak protein band above $95 \mathrm{kDa}$ ) and in the cytosolic fraction (protein bands at $\sim 70$, 75 , and $125 \mathrm{kDa}$ ). A control treatment with wild-type Cdt resulted in the localization of $\mathrm{CdtB}$ mainly in the nuclear fraction (Figure 4b). A further control treatment with PA+ FP59AGG located FP59AGG as shown for LFnCdtB in the nuclear and the cytosolic fractions (Figure 4c).

Nuclear activity of LFnCdtB was detected by immunofluorescence detection of phosphorylated form of histone $\mathrm{H} 2 \mathrm{~A}$.X ( $\mathrm{pH} 2 \mathrm{~A} . \mathrm{X})$ as a marker of DNA damage. HeLa cells treated with PA + LFnCdtB or treated with wild-type Cdt showed a clear increase in H2A.X phosphorylation after $8 \mathrm{~h}$ (Figure $4 \mathrm{~d}$ ). The signal remained elevated at $24 \mathrm{~h}$, but appeared slightly reduced for $\mathrm{PA}+\mathrm{LFnCdtB}$ treatment, possibly a result of induced apoptosis. Treatment with PA + FP59AGG resulted in a very weak increase of phosphorylation compared with PA + LFnCdtB treatment. This could also be a side effect of apoptosis induction in FP59AGG-treated cells.

Tumor treatment. LFnCdtB was injected intraperitoneally together with either the matrix metalloproteinase-activated PA-L1 or with wild-type PA into mice having implanted LL3 mouse melanoma cell tumors. Treatment with PA-L1+ LFnCdtB was well tolerated, resulting in no significant change in body weight, whereas the PA + LFnCdtB group suffered a nearly $20 \%$ body weight loss by day 12 


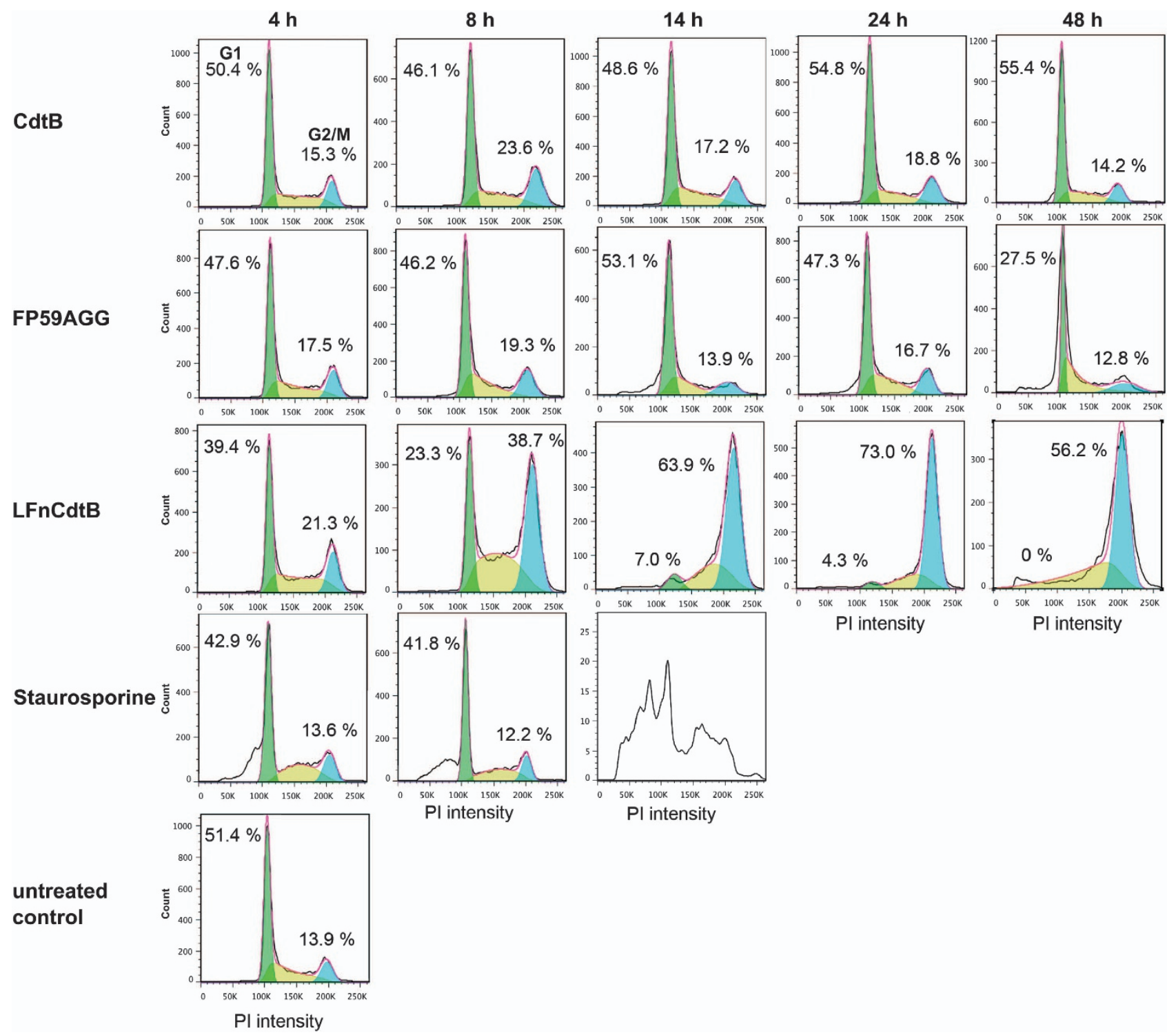

Figure 2 Cell cycle analysis of CHO K1 cells $\left(0.2 \times 10^{6}\right.$ cells) treated with $250 \mathrm{ng} / \mathrm{ml} \mathrm{PA}$ and $100 \mathrm{pM} \mathrm{CdtB}$ or LFnCdtB or $0.1 \mathrm{pM} \mathrm{FP59AGG} \mathrm{for} \mathrm{4-48} \mathrm{h.} \mathrm{As} \mathrm{a} \mathrm{control,} \mathrm{cells}$ were incubated with $1 \mu \mathrm{M}$ staurosporine for $4-14 \mathrm{~h}$ to induce apoptosis. Cells were fixed with ethanol, stained by propidium iodide to quantify cellular DNA, and 25000 cells were counted by flow cytometry. Cell cycle analysis by FlowJo software (Flow Jo version 7.6; Tree Star, Ashland, OR, USA) indicated the percentage of cells in the G0/G1 phase (green cell population and percentage on the left side in each panel) and in the G2/M phase (turquois cell population and percentage on the right side in each panel)

(Figure 5a), and two mice were found dead on day 12. Analysis of blood enzymes showed no increase of alanine aminotransferase and lactate dehydrogenase and mild increases of aspartate aminotransferase and creatine kinase at the end of the treatment regimen with PA-L1 and LFnCdtB (Supplementary Figure S4). Although PA + LFnCdtB treatment inhibited tumor growth as observed on day 12 (Figure $5 b$ ), these mice were euthanized on day 13 because of the substantial weight loss, and thus only received three injections of PA + LFnCdtB (days 5, 7, and 9). Mice in the PBS-treated control group showed rapid tumor growth and ulceration, and were euthanized on day 19. In contrast, PA-L1 + LFnCdtB treatment resulted in strong tumor growth inhibition (Figure 5b). One mouse treated with PA-L1+ LFnCdtB died on day 23, 7 days after the last injection with
PA-L1 + LFnCdtB. No apparent side effects of the treatment were observed and the cause of death remained unclear. At this time this mouse was tumor-free (by palpation). On day 19 ( 3 days after the last injection) 5 of 10 mice of the PA-L1 + LFnCdtB group were tumor-free. Without further toxin injections, tumor sizes in three mice decreased further; thus, eight out of nine surviving mice of the PA-L1 + LFnCdtB group were tumor-free on day 28 and remained tumor-free for the next 2 months. The mouse with the remaining tumor was euthanized after 2 weeks due to tumor regrowth.

\section{Discussion}

The modified toxin used in our study utilizes the highly efficient anthrax toxin delivery system which can deliver a number of 


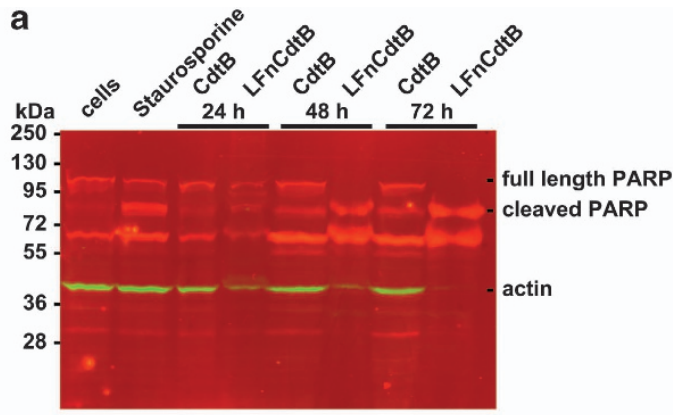

b

CdtB

FP59AGG

LFnCdtB
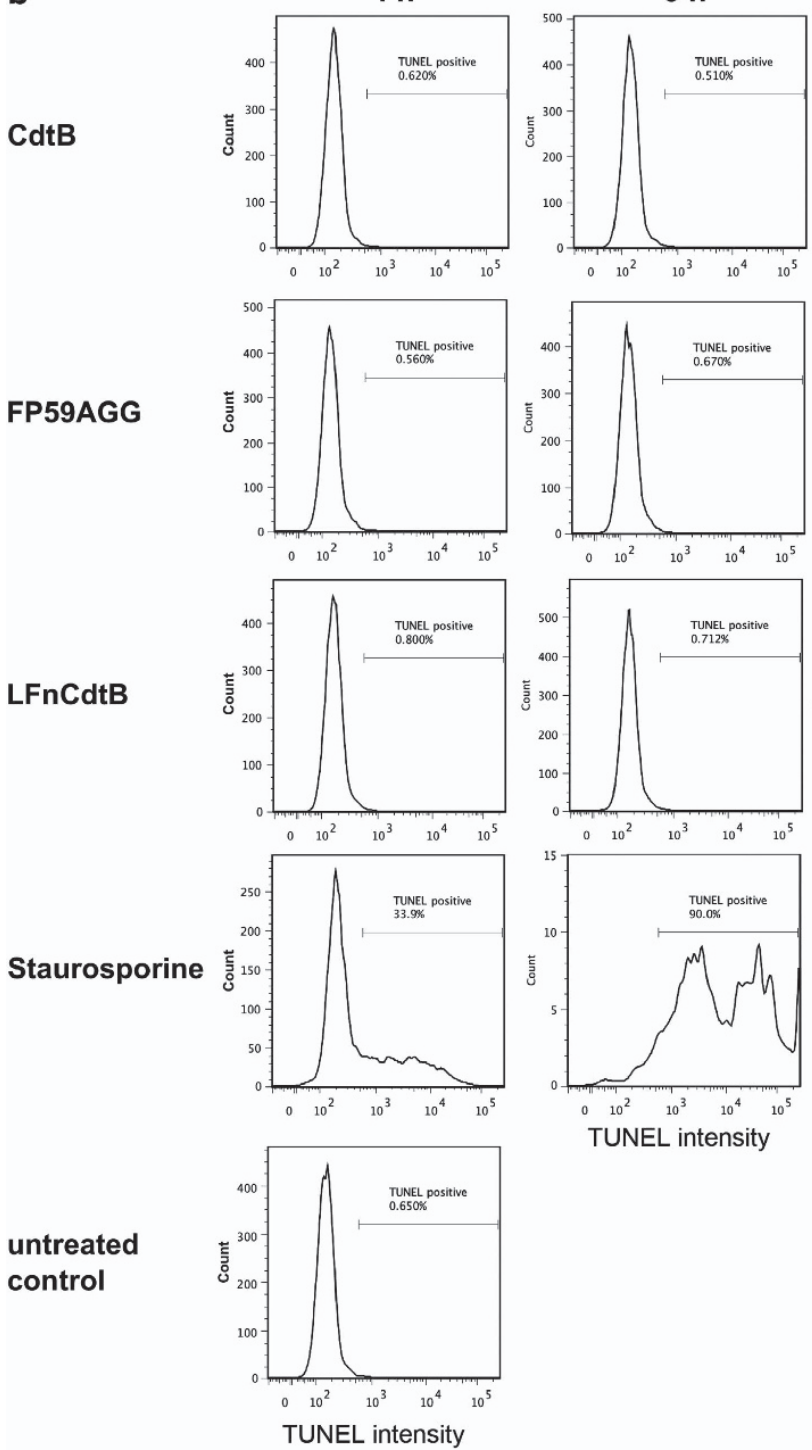

$14 \mathrm{~h}$
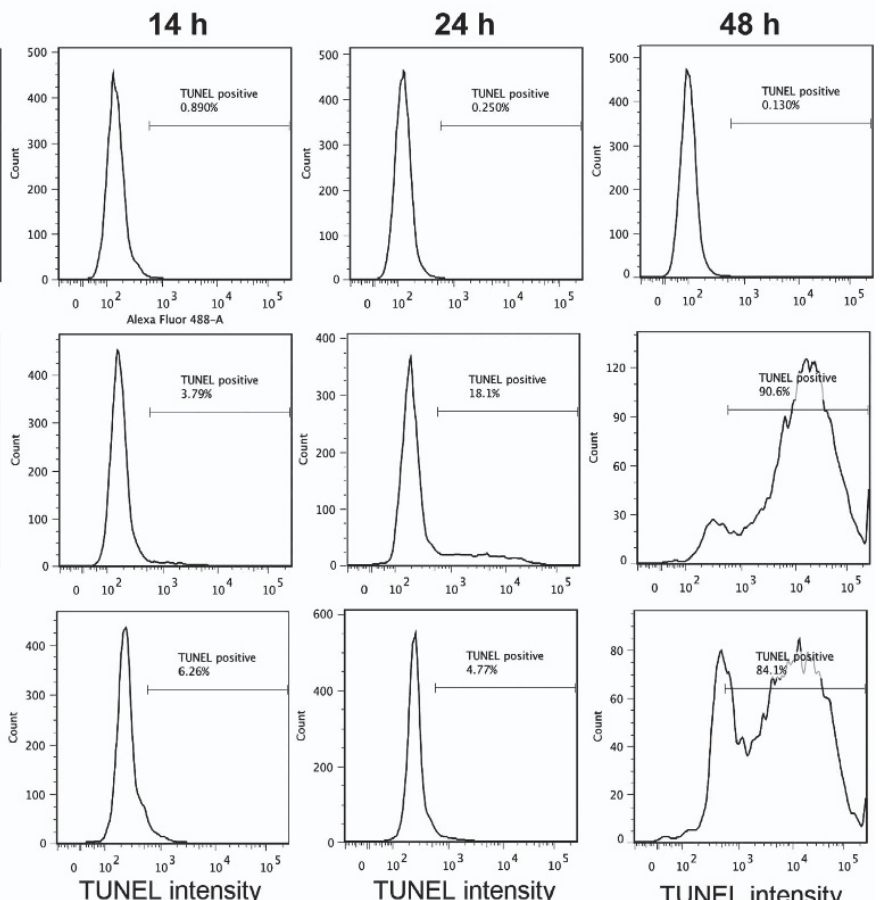

TUNEL intensity

Figure 3 Apoptosis induction by LFnCdtB analyzed by PARP cleavage (a) and TUNEL staining (b). (a) HeLa cells $\left(1 \times 10^{6}\right.$ cells) were exposed to $250 \mathrm{ng} / \mathrm{ml}$ PA and $10 \mathrm{nM} \mathrm{LFnCdtB}$ or CdtB for $24-72 \mathrm{~h}$. As a control, cells were incubated with $1 \mu \mathrm{M}$ staurosporine for $2 \mathrm{~h}$ to induce apoptosis. Cells were lyzed and analyzed by simultaneous anti-PARP (red signals) and anti-actin (green signals) immunodetection following western blotting. (b) $\mathrm{CHO}$ K1 cells $\left(0.2 \times 10^{6}\right.$ cells) were treated with $250 \mathrm{ng} / \mathrm{ml}$ PA and $100 \mathrm{pM}$ CdtB or LFnCdtB or $0.1 \mathrm{pM} \mathrm{FP59AGG}$ for $4-48 \mathrm{~h}$. As a control, cells were incubated with $1 \mu \mathrm{M}$ staurosporine for $4-14 \mathrm{~h}$ to induce apoptosis. Cells were fixed with ethanol, stained by TUNEL staining to quantify apoptotic cells, and 10000 cells were counted by flow cytometry. TUNEL-positive apoptotic cells were gated by high fluorescence and the percentage is indicated in every panel

different 'payloads' in addition to the natural toxin LF, as it was shown that the system is able to deliver Pseudomonas exotoxin A catalytic domain, ${ }^{14}$ diphtheria toxin A chain, shiga toxin catalytic domain, ${ }^{15} \mathrm{Bcl}-\mathrm{XL}$ protein, ${ }^{16}$ ricin toxin $\mathrm{A}$ chain, ${ }^{17}$ the reporter beta-lactamase, ${ }^{18,19}$ and flagellin. ${ }^{20}$ Wild-type PA binds to either one of its two cell surface 


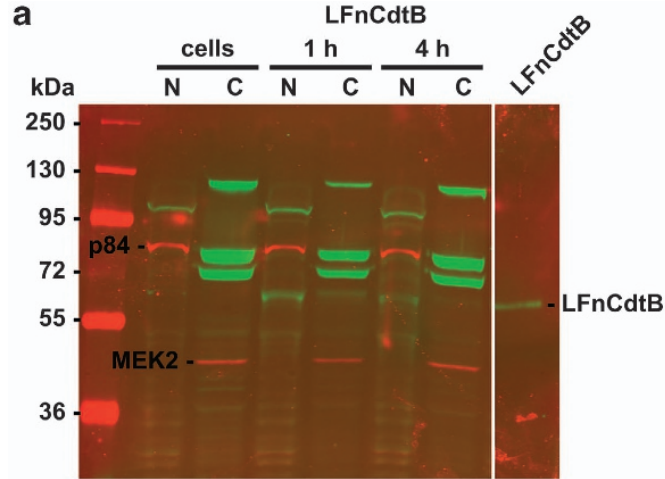

d

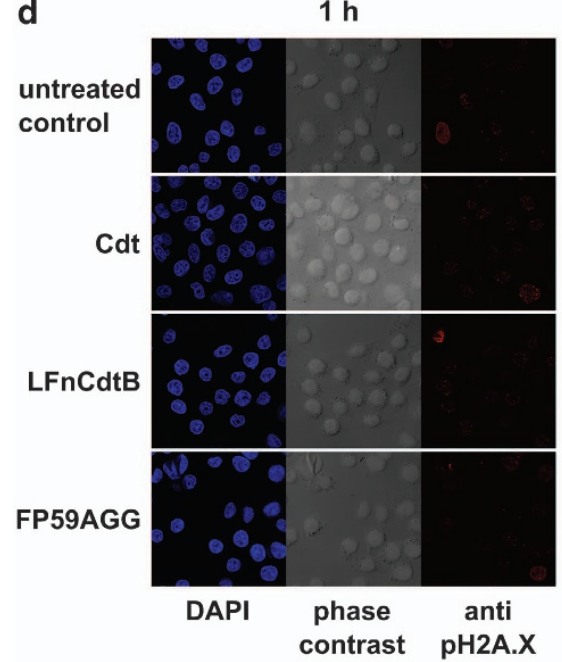

b

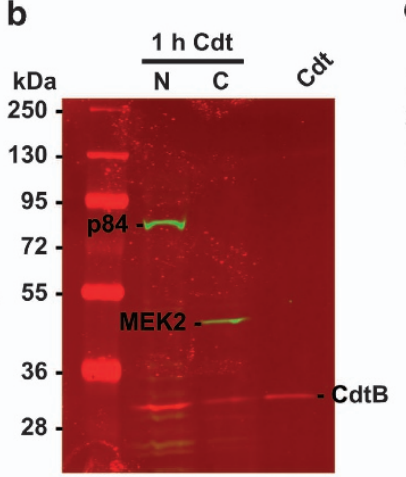

$8 \mathrm{~h}$

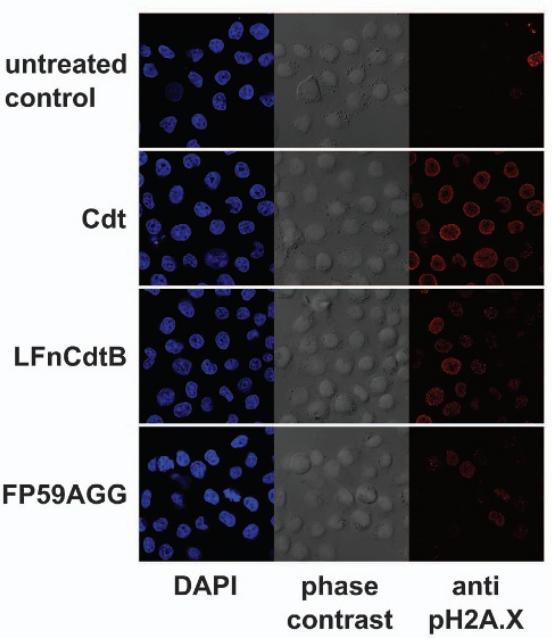

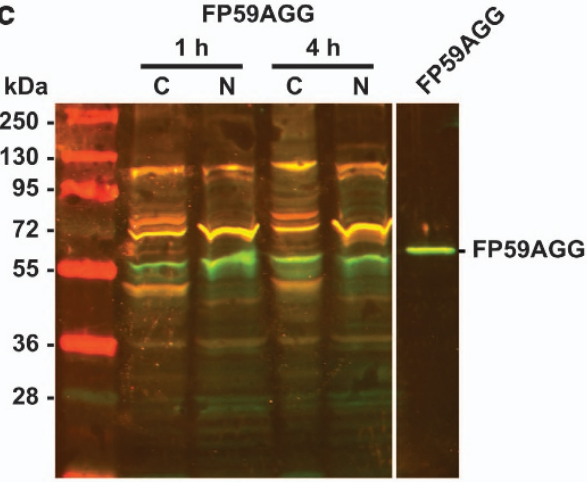

$24 \mathrm{~h}$

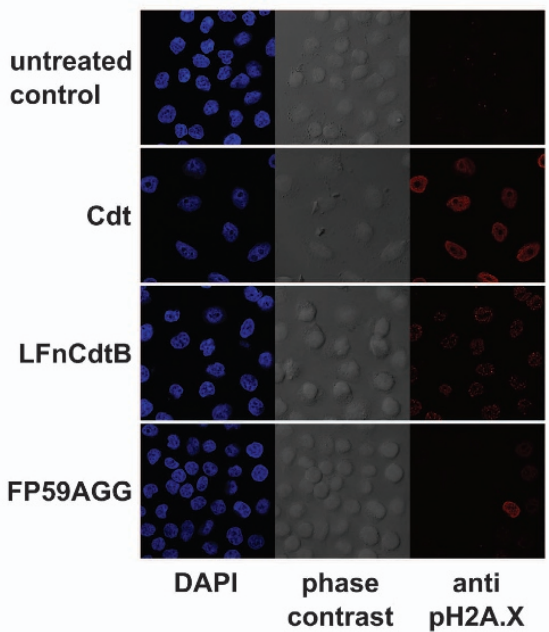

Figure 4 Intracellular detection (a-c) and nuclear action (d) of LFnCdtB. (a-c) To detect the fusion proteins in the cytosolic or nuclear fraction of cells, CHO K1 cells $\left(1.75 \times 10^{6} \mathrm{cells}\right)$ were incubated with $250 \mathrm{ng} / \mathrm{ml} \mathrm{PA}$ and $5 \mathrm{nM}$ biotinylated LFnCdtB or FP59AGG for 1 or $4 \mathrm{~h}$. Additional cells were incubated with a mixture of $100 \mathrm{nM}$ each $\mathrm{CdtA}, \mathrm{CdtB}$, and $\mathrm{CdtC}(\mathrm{Cdt})$. Cytosolic and nuclear fractions were isolated, and $40 \mu \mathrm{g}$ total protein of each fraction were separated by SDS-PAGE and submitted to western blotting. Immunodetection was performed simultaneously by streptavidin (a, green signal), anti-CdtB (b, red signal), anti-LF (c, red signal), and anti-Pseudomonas exotoxin A (c, green signal), and anti-p84 (nuclear marker protein, red signal in a and green signal in $\mathbf{b}$ ) and anti-MEK2 (cytosolic marker protein, red signal in a and green signal in $\mathbf{b}$ ). Merged signals are shown in yellow. The nuclear action of CdtB was detected by immunofluorescence detection of phosphorylated histone H2A.X (d). HeLa cells $\left(0.2 \times 10^{6}\right.$ cells on cover slips) were incubated with $250 \mathrm{ng} / \mathrm{ml} \mathrm{PA}$ and $10 \mathrm{nM} \mathrm{LFnCdtB}$ or $10 \mathrm{nM} \mathrm{FP59AGG} \mathrm{or} \mathrm{with} 100 \mathrm{nM}$ CdtA, CdtB, and CdtC each (Cdt) for 1-24 h. Cells were fixed and permeabilized before staining by anti-pH2A.X and DAPI. Results show DAPI fluorescence in blue (nuclear DNA, left panel), phase contrast (middle panel), and pH2A.X fluorescence in red (DNA damage, right panel)

receptors, of which capillary morphogenesis gene 2 is expressed on nearly all cell types. ${ }^{21}$ This allows for the targeting of nearly all tissues, including tumor cells. Therefore, the combination of PA + FP59AGG efficiently inhibited the growth of all tumor cell lines tested in this study (Table 1). Protein synthesis inhibition resulted in induction of apoptosis and cell death. Very few types of tumor cells evade killing by toxins that block protein synthesis. ${ }^{22}$ Mutation of the furin protease cleavage site within PA to sequences recognized by either urokinase plasminogen activator or matrix metalloproteinase-2 renders PA a highly valuable tool for targeting certain tumor cells. ${ }^{23}$ Both LF (as an anti-angiogenic drug) and FP59 have been used successfully in tumor therapy in preclinical studies. ${ }^{24,25}$ In 2005 , Liu et al. ${ }^{26}$ described a method for dual proteinase-specificity using the PA delivery system. This system was recently improved and resulted in the development of a system that requires both urokinase plasminogen activator and matrix metalloproteinase-2 activity. ${ }^{25}$
CdtB from $A$. actinomycetemcomitans has been described as a metal-dependent endonuclease with an activity $\sim 1000-$ fold lower than bovine DNase $1,{ }^{27}$ and Shenker et al. ${ }^{28}$ reported that CdtB has the same phosphatase activity as that of PTEN; thus, the precise mechanism for toxicity remains uncertain. Previous studies have attempted to use Cdt for the targeting of cancer cells. For example, a mutant of CdtA (unable to bind its natural receptor) from $A$. actinomycetemcomitans has been conjugated to a human monoclonal antibody against CD133 to target the toxin to CD133 + head and neck cancer cell lines, achieving modest success in growth inhibition of CD133+ tumor cells. ${ }^{29}$ Another study described the use of CdtB fused to high-affinity $\lg E$ to target and degranulate mast cells. ${ }^{30}$ This study used CdtB without the natural translocation mechanism described for $\mathrm{Cdt}$ (which includes CdtA and CdtC), which may affect the efficacy of the treatment. It is currently debated how CdtB translocates to the nucleus of host cells. Damek-Poprawa et al. ${ }^{31}$ reported the retention of the $A$ subunit at the cell surface and the direct 

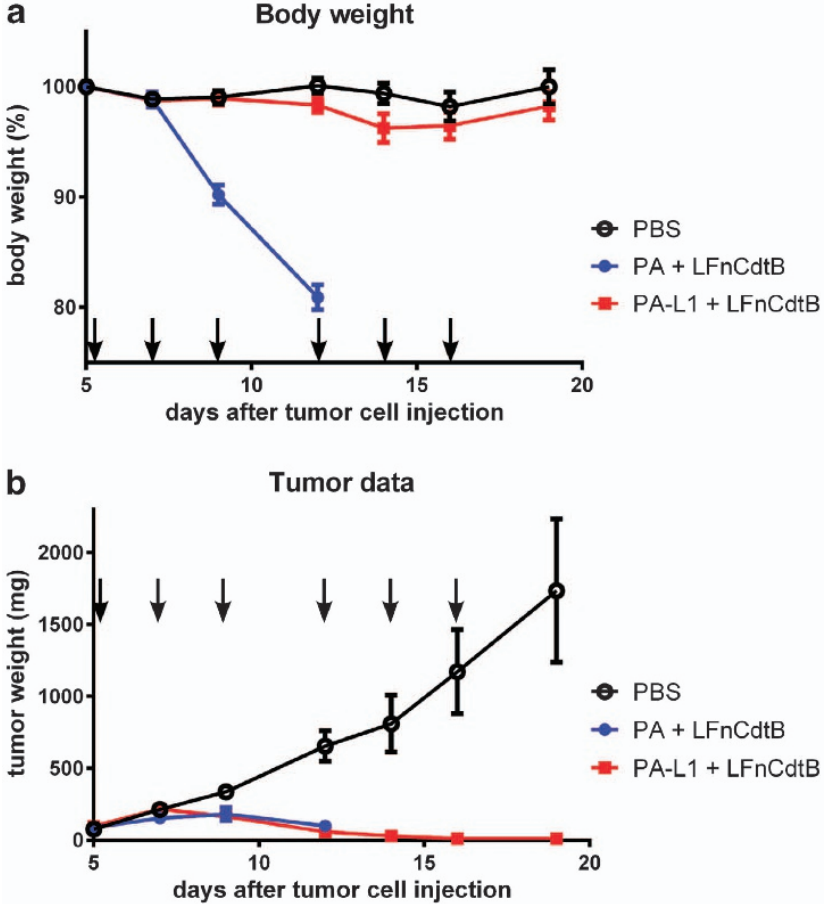

Figure 5 Tumor treatment by LFnCdtB in a mouse model. 10-13 C57BL/6 mice per group were injected subcutaneously with LL3 mouse melanoma tumors on day 0 . Injection of PA + LFnCdtB or tumor-specific PA-L1 + LFnCdtB fusion into the mouse peritoneum was performed on days 5, 7, 9, 12, 14, and 16 (arrows) using either PBS (black line), $100 \mu \mathrm{g}$ wild-type PA $+100 \mu \mathrm{g}$ LFCdtB (blue line) or $100 \mu \mathrm{g}$ $\mathrm{PA}-\mathrm{L} 1+100 \mu \mathrm{g} \mathrm{LFnCdtB}$ (red line). (a) Relative mean mouse body weight until day 19. Error bars indicate the S.D. (b) Mean tumor masses until day 19. Tumor mass was calculated from measurements of the tumor width, depth, and height

delivery of the $B$ subunit from the endoplasmic reticulum to the nucleus, most likely by the chaperoning action of the $\mathrm{C}$ subunit. Our approach of using PA + LFn for the delivery of CdtB to the cytosol of a targeted cell circumvents these uncertainties about the trafficking of the native toxin. Instead, we use the highly efficient and flexible targeting system provided by the anthrax toxin proteins to deliver the enzymatic CdtB subunit to the cytosol of cells. LFnCdtB may still be small enough to diffuse from the cytosol to the nucleus, its presumed site of action, or it may contain a nuclear localization signal that helps directing LFnCdtB to the nucleus. Such a signal was identified in $\mathrm{CdtB}$ of $A$. actinomycetemcomitans, which shares a very high protein sequence homology (97\% identical amino acids ${ }^{32}$ ) with CdtB from $\mathrm{H}$. ducreyi used in this study. ${ }^{33}$ Nevertheless, our data demonstrate that LFnCdtB was successfully delivered to the cytosol within $1 \mathrm{~h}$ of toxin exposure, and that it reached the nucleus (Figure 4a). Furthermore, its activity was preserved, evidenced by the phosphorylation of histone H2A.X (Figure 4d), by induction of the cell cycle arrest in the G2/M phase (Figure 2).

In mouse tumor studies, the combination of the matrix metalloproteinase-2-specific PA-L1 + LFnCdtB revealed a surprisingly low toxicity. Six bolus injections of $100 \mu \mathrm{g}$ (2.3-4.3 $\mathrm{mg} / \mathrm{kg}$ body weight) of both proteins every other day into the mouse peritoneum did not result in any apparent side effects as observed by monitoring the body weight and the behavior of the mice. Analysis of blood enzymes showed a mild increase in two out of the four measured parameters (aspartate aminotransferase and creatine kinase, Supplementary Figure S4), indicating some damage to the tissue. Liver damage is unlikely, as alanine aminotransaminase levels were not elevated. Elevated levels of aspartate aminotransferase and creatine kinase indicate some myocardial tissue damage. However, blood samples were analyzed $1 \mathrm{~h}$ after the last injection of the 2-week treatment regimen and a time course of blood enzyme levels would help to understand the relevance of the reported enzyme levels. The $100 \mu \mathrm{g}$ dose is an extremely high tolerable dose compared with similar drugs. Previous studies on PA-L1 + LF identified only $45 \mu \mathrm{g} \mathrm{PA}-\mathrm{L} 1$ and $15 \mu \mathrm{g} \mathrm{LF}$ as the maximal tolerated dose. ${ }^{1}$ The dual protease system described earlier (depending on both urokinase plasminogen activator and matrix metalloproteinase-2 activity) was the safest system so far with a maximal tolerated dose of slightly below $25 \mu \mathrm{g}$ of each protein component, given as six injections on alternate days. ${ }^{25}$ Other targeted toxins showed even higher non-specific toxicities than the sophisticated tumor-targeted anthrax toxin system. A fusion protein containing the protein toxin saporin fused to epidermal growth factor was injected only three times subcutaneously at a maximal dose of $10 \mu \mathrm{g} /$ injection. ${ }^{34}$

The data presented here demonstrate the successful cellular delivery of a fusion of LFn and CdtB to various tumor cells by the anthrax toxin delivery system (PA and LFn). The combination with tumor-specific PA resulted in the design of a highly efficient antitumor drug that eliminates tumor cells by damaging nuclear DNA, inducing cell cycle arrest and subsequently apoptosis. Animal experiments demonstrated the great efficiency of this drug by curing $90 \%$ of mice from growing tumors without inducing any observable side effects. Future studies will provide further validation on the suitability of this new drug system for safe and efficient elimination of tumors in different tumor models and, eventually, may warrant clinical trials in humans.

\section{Materials and Methods}

Cloning of LFnCdtB. LFnCdtB consists of the N-terminal 255 residues of anthrax toxin $L F$, and has the sequence AGG...NLS. LFn is fused C-terminally via an 11-residue linker to the 261-residue $\mathrm{CdtB}$, which has the sequence NLSD...VRDR. Details on cloning, expression, and purification of LFnCdtB are described in the Supplementary Information (Supplementary Text S1). FP59AGG, wild-type PA, and tumor-specific PA-L1 were expressed and purified from avirulent strains of $B$. anthracis by methods described earlier. ${ }^{35}$ Recombinant CdtA, CdtB, and CdtC were kindly supplied by Jerry Keith (National Institute of Child Health and Human Disease, NIH, Bethesda, MD, USA).

Cell culture and cytotoxicity assays. Cell culture experiments were performed using HeLa cells (human cervical carcinoma cell line), CHO K1 cells (Chinese hamster ovary cells), HN6 cells (human head and neck cancer cell line), RAW264.7 cells (murine leukemic monocyte/macrophages), LL3 cells (murine Lewis Lung carcinoma cells), B16/BL6 (murine melanoma cells), and T241 (murine fibrosarcoma cells). An additional 14 human cell lines from the NCl-60 panel ${ }^{36}$ used in certain experiments included melanoma cells (C32, Malme-3M, SK-MEL-2, SK-MEL-24, and SK-MEL-28), colon carcinoma cells (COLO 205, HCC2998, and SW620), lung carcinoma (A549 and NCl-H226), renal carcinoma (A-498 and SC12C), and mamma carcinoma (MDA-MB-231 and Hs 578T). See Supplementary Information for details on cell culture and cytotoxicity assays (Supplementary Text S1).

Cell cycle inhibition and TUNEL assay. $\mathrm{CHO} \mathrm{K1}$ cells $\left(0.2 \times 10^{6}\right.$ cells per well, grown overnight in six-well plates) were incubated with $250 \mathrm{ng} / \mathrm{ml} \mathrm{PA}$ and 
$100 \mathrm{pM}$ CdtB or LFnCdtB or $0.1 \mathrm{pM}$ FP59AGG in $800 \mu \mathrm{l}$ cell culture medium for $1-48 \mathrm{~h}$. Similar cultures were incubated with $1 \mu \mathrm{M}$ staurosporine as a positive control for the TUNEL assay. Cells were trypsinized and resuspended in $1 \mathrm{ml}$ cell culture medium, centrifuged $\left(5 \mathrm{~min}, 200 \times \mathrm{g}, 4^{\circ} \mathrm{C}\right)$ and resuspended in $1 \mathrm{ml} \mathrm{PBS}$. Cold $100 \%$ ethyl alcohol was added dropwise under constant mixing to a final concentration of $70 \%$ to fix the cells, and cells were either incubated 15 min on ice or overnight at $-20^{\circ} \mathrm{C}$. For cell cycle analysis by propidium iodide staining, the fixed cells were centrifuged $\left(5 \mathrm{~min}, 430 \times \mathrm{g}, 4^{\circ} \mathrm{C}\right)$ and resuspended in $500 \mu \mathrm{l}$ staining solution ( $50 \mu \mathrm{g} / \mathrm{ml}$ propidium iodide (Life Technologies, Grand Island, NY, USA), $0.1 \mathrm{mg} / \mathrm{ml}$ RNase A (Qiagen, Valencia, CA, USA), 0.05\% Triton X-100). After incubation for $40 \mathrm{~min}$ at $37^{\circ} \mathrm{C}, 3 \mathrm{ml}$ PBS was added, the cells centrifuged ( $5 \mathrm{~min}, 430 \times \mathrm{g}, 4^{\circ} \mathrm{C}$ ), $3 \mathrm{ml}$ supernatant removed, and cellular fluorescence was measured by flow cytometry on an LSR II (BD Biosciences, San Jose, CA, USA) using the Texas Red channel and counting 25000 cells. Detection of DNA damage with the TUNEL assay was performed on cells treated and fixed as described above. TUNEL staining was performed with the APO-BrdU TUNEL Assay Kit (Life Technologies) according to the manufacturer's instructions. Cellular fluorescence was determined by flow cytometry on an LSR II (BD Biosciences), counting 10000 cells.

\section{Intracellular detection of LFn fusion proteins and PARP} cleavage. For the intracellular detection of LFn fusion proteins, $\mathrm{CHO} \mathrm{K1}$ cells $\left(1.75 \times 10^{6}\right.$ cells per well, grown overnight in six-well plates) were incubated with $250 \mathrm{ng} / \mathrm{ml} \mathrm{PA}$ and $5 \mathrm{nM} \mathrm{LFnCdtB}$ or FP59AGG in $800 \mu \mathrm{l}$ medium for 1 or $4 \mathrm{~h}$. The LFnCdtB used in this analysis was biotinylated using $(+)$-Biotin N-hydroxysuccinimide ester (Sigma, St. Louis, MO, USA). In parallel, cells were incubated for $4 \mathrm{~h}$ with a mixture of $100 \mathrm{nM}$ each of $\mathrm{CdtA}, \mathrm{CdtB}$, and $\mathrm{CdtC}(\mathrm{Cdt})$. After the indicated incubation time, cytosolic fractions and nuclear fractions were isolated by using the Nuclear Extract Kit (Active Motif, Carlsbad, CA, USA) according to the manufacturer's instructions. Total protein concentrations were determined by using the ProStain assay (Active Motif), and $40 \mu \mathrm{g}$ of total protein was separated by SDS-PAGE and western blotted using the iBlot system (Life Technologies) and the Western Blot Signal Enhancer kit (Thermo, Waltham, MA, USA). For apoptosis detection, HeLa cells $\left(1 \times 10^{6}\right.$ cells per well, grown overnight in six-well plates) were incubated with $250 \mathrm{ng} / \mathrm{ml}$ PA and $10 \mathrm{nM} \mathrm{LFnCdtB}$ or CdtB in $800 \mu$ medium for 24-72 $\mathrm{h}$. In parallel, cells were incubated with $1 \mu \mathrm{M}$ staurosporine for $2 \mathrm{~h}$. Cells were washed and scraped off in $100 \mu \mathrm{l}$ PBS $+1 \%$ Triton X-100, incubated $30 \mathrm{~min}$ at $4^{\circ} \mathrm{C}$ on a rotary shaker and centrifuged $\left(30 \mathrm{~min}, 16000 \times \mathrm{g}, 4^{\circ} \mathrm{C}\right)$. Supernatants were further incubated for $7 \mathrm{~h}$ at $37^{\circ} \mathrm{C}$, total protein concentrations were determined by the BCA assay (Thermo Fisher Scientific, Rockford, IL, USA), and $100 \mu \mathrm{g}$ of total protein was separated by SDS-PAGE and western blotted using the iBlot system (Life Technologies). For intracellular localization, cytosolic marker protein MEK2 and nuclear marker protein p84 were detected by antibodies (anti-MEK2 (N-20), rabbit polyclonal IgG sc-524, Santa Cruz, Dallas, TX, USA; anti-p84, monoclonal rabbit IgG ab131268, Abcam, Cambridge, MA, USA). Detection of toxin proteins was achieved with rabbit anti-LF and mouse anti-CdtB, both made in our laboratory, and goat anti-Pseudomonas exotoxin A (List Biological Laboratories, Campbell, CA, USA). Apoptosis induction was detected by the use of anti-PARP polyclonal antibodies (BD Biosciences). All primary antibodies were detected by infrared dye-conjugated secondary antibodies on the Odyssey Imager infrared detection system (LI-COR, Lincoln, NE, USA).

pH2A.X assay. HeLa cells $\left(0.2 \times 10^{6}\right.$ cells per well, grown overnight in 24-well plates on cover slips) were incubated for $1-24 \mathrm{~h}$ with $250 \mathrm{ng} / \mathrm{ml}$ PA and $10 \mathrm{nM}$ LFnCdtB or $10 \mathrm{nM}$ FP59AGG, or with $100 \mathrm{nM}$ Cdt. Cells were fixed and permeabilized by incubation with $4 \%$ paraformaldehyde and $0.1 \%$ Triton X-100 in PBS for $15 \mathrm{~min}$, followed by blocking with PBS $+0.05 \%$ Tween- $20+1 \%$ bovine serum albumin and by treatment with antibodies against phosphorylated histone H2A.X (pH2A.X, Cell Signaling, Danvers, MA, USA) for $2 \mathrm{~h}$. Bound antibodies were detected by the use of Alexa-Fluor488-conjugated secondary antibodies; cellular DNA was stained with 4',6-diamidino-2-phenylindole (DAPI, $1 \mu \mathrm{g} / \mathrm{ml}$ in PBS). Images of mounted cover slips were taken by confocal laser scanning microscopy on a SP5 microscope (Leica, Buffalo Grove, IL, USA).

Animal experiments. All animal experiments were performed under protocols approved by the NIAID Animal Care and Use Committee. Both male and female C57BL/6 mice (3 to 6 months old, Jackson Laboratories, Bar Harbor, $\mathrm{ME}$, USA) were subcutaneously injected in the neck with $0.8 \times 10^{6}$ LL3 mouse melanoma cells on day 0 . After 5 days (all mice had tumors with a width of a least
$4 \mathrm{~mm}$ ) mice were randomly assigned to three groups of 10 or 13 mice each and injected every other day (days $5,7,9,12,14$, and 16) intraperitoneally with $500 \mu \mathrm{l}$ sterile PBS containing different drug combinations: PBS (13 mice), $100 \mu \mathrm{g}$ wildtype PA $+100 \mu \mathrm{g}$ LFnCdtB in PBS (10 mice), or $100 \mu \mathrm{g}$ PA-L1 (PA with a mutated furin cleavage site to achieve tumor-selective cleavage and activation of PA by matrix metalloproteinase- $\left.2^{11}\right)+100 \mu \mathrm{g} \mathrm{LFnCdtB}$ in PBS (10 mice). Tumors and mouse body weight were measured every other day with a caliper, and tumor masses calculated (tumor mass $(\mathrm{mg})=$ (width (all in $\mathrm{mm}) \times$ depth $\times$ height)/2). Mice having tumors with a diameter exceeding $20 \mathrm{~mm}$ or ulceration or having more than $20 \%$ body weight loss were euthanized according to the animal protocol. For blood enzyme measurements, the blood was collected $1 \mathrm{~h}$ after the last injection (same treatment regimen described above, five mice treated with PBS, five mice treated with $100 \mu \mathrm{g} \mathrm{PA}-\mathrm{L} 1+100 \mu \mathrm{g} \mathrm{LFnCdtB}$ ), and the plasma was prepared to measure alanine aminotransferase, aspartate aminotransferase, lactate dehydrogenase, and creatine kinase in the National Institutes of Health, Clinical Center.

\section{Conflict of Interest}

Dr. Leppla and Dr. Bachran are inventors of a pending patent application described in this work. The remaining authors declare no conflict of interest.

Acknowledgements. This research was supported by the Intramural Research Program of the $\mathrm{NIH}$, National Institute of Allergy and Infectious Diseases (NIAID), Bethesda, MD, USA. We thank Suzanne Abdelazim for her support, Eric Anderson (NIDDK) for mass spectrometric analysis of the proteins described here, Margery Smelkinson (NIAID) and Owen Schwartz (NIAID) for confocal laser scanning microscopy analyses and Kevin Holmes (NIAID) for help with flow cytometry analyses.

1. Liu S, Wang H, Currie BM, Molinolo A, Leung $\mathrm{HJ}$, Moayeri $\mathrm{M}$ et al. Matrix metalloproteinase-activated anthrax lethal toxin demonstrates high potency in targeting tumor vasculature. J Biol Chem 2008; 283: 529-540.

2. Kintzer AF, Thoren KL, Sterling HJ, Dong KC, Feld GK, Tang II et al. The protective antigen component of anthrax toxin forms functional octameric complexes. J Mol Biol 2009; 392: 614-629.

3. Wising C, Molne L, Jonsson IM, Ahlman K, Lagergard T. The cytolethal distending toxin of Haemophilus ducreyi aggravates dermal lesions in a rabbit model of chancroid. Microbes Infection/Institut Pasteur 2005; 7: 867-874.

4. Lagergard T, Lundqvist A, Wising C, Gabrielsson V, Ahlman K. Formaldehyde treatment increases the immunogenicity and decreases the toxicity of Haemophilus ducreyi cytolethal distending toxin. Vaccine 2007; 25: 3606-3614.

5. Anderson JD, MacNab AJ, Gransden WR, Damm SM, Johnson WM, Lior H. Gastroenteritis and encephalopathy associated with a strain of Escherichia coli 055:K59:H4 that produced a cytolethal distending toxin. Pediatr Infect Dis J 1987; 6: $1135-1136$.

6. Carette JE, Guimaraes CP, Varadarajan M, Park AS, Wuethrich I, Godarova A et al. Haploid genetic screens in human cells identify host factors used by pathogens. Science 2009; 326: 1231-1235.

7. Elwell CA, Dreyfus LA. DNase I homologous residues in CdtB are critical for cytolethal distending toxin-mediated cell cycle arrest. Mol Microbiol 2000; 37: 952-963.

8. Jinadasa RN, Bloom SE, Weiss RS, Duhamel GE. Cytolethal distending toxin: a conserved bacterial genotoxin that blocks cell cycle progression, leading to apoptosis of a broad range of mammalian cell lineages. Microbiology 2011; 157(Pt 7): 1851-1875.

9. Shenker BJ, Dlakic M, Walker LP, Besack D, Jaffe E, LaBelle E et al. A novel mode of action for a microbial-derived immunotoxin: the cytolethal distending toxin subunit $B$ exhibits phosphatidylinositol 3,4,5-triphosphate phosphatase activity. J Immunol 2007; 178: 5099-5108.

10. Pomerantsev AP, Pomerantseva OM, Moayeri M, Fattah R, Tallant C, Leppla SH. A Bacillus anthracis strain deleted for six proteases serves as an effective host for production of recombinant proteins. Protein Expr Purif 2011; 80: 80-90.

11. Liu S, Netzel-Arnett S, Birkedal-Hansen H, Leppla SH. Tumor cell-selective cytotoxicity of matrix metalloproteinase-activated anthrax toxin. Cancer Res 2000; 60: 6061-6067.

12. Liu S, Wang H, Currie BM, Molinolo A, Leung HJ, Moayeri $M$ et al. Matrix metalloproteinase-activated anthrax lethal toxin demonstrates high potency in targeting tumor vasculature. J Biol Chem 2008; 283: 529-540.

13. Chandler CS, Ballard FJ. Multiple biotin-containing proteins in 3T3-L1 cells. Biochem J 1986; 237: 123-130.

14. Arora N, Leppla SH. Residues 1-254 of anthrax toxin lethal factor are sufficient to cause cellular uptake of fused polypeptides. J Biol Chem 1993; 268: 3334-3341. 
15. Arora N, Leppla SH. Fusions of anthrax toxin lethal factor with shiga toxin and diphtheria toxin enzymatic domains are toxic to mammalian cells. Infect Immun 1994; 62: 4955-4961.

16. Liu XH, Collier RJ, Youle RJ. Inhibition of axotomy-induced neuronal apoptosis by extracellular delivery of a Bcl-XL fusion protein. J Biol Chem 2001; 276: 46326-46332.

17. McCluskey AJ, Olive AJ, Starnbach MN, Collier RJ. Targeting HER2-positive cancer cells with receptor-redirected anthrax protective antigen. Mol Oncol 2012; 7: 440-451.

18. Hobson JP, Liu S, Rono B, Leppla SH, Bugge TH. Imaging specific cell-surface proteolytic activity in single living cells. Nat Met 2006; 3: 259-261.

19. Hu H, Leppla SH. Anthrax toxin uptake by primary immune cells as determined with a lethal factor-beta-lactamase fusion protein. PLOS One 2009; 4: e7946.

20. von Moltke J, Trinidad NJ, Moayeri M, Kintzer AF, Wang SB, van Rooijen N et al. Rapid induction of inflammatory lipid mediators by the inflammasome in vivo. Nature 2012; 490 : 107-111.

21. Liu S, Crown D, Miller-Randolph S, Moayeri M, Wang $\mathrm{H}, \mathrm{Hu} \mathrm{H}$ et al. Capillary morphogenesis protein-2 is the major receptor mediating lethality of anthrax toxin in vivo. Proc Natl Acad Sci USA 2009; 106: 12424-12429.

22. Traini R, Ben-Josef G, Pastrana DV, Moskatel E, Sharma AK, Antignani A et al. ABT-737 overcomes resistance to immunotoxin-mediated apoptosis and enhances the delivery of pseudomonas exotoxin-based proteins to the cell cytosol. Mol Cancer Therap 2010; 9 : 2007-2015

23. Liu S, Bugge TH, Leppla SH. Targeting of tumor cells by cell surface urokinase plasminogen activator-dependent anthrax toxin. J Biol Chem 2001; 276: 17976-17984.

24. Liu S, Aaronson H, Mitola DJ, Leppla SH, Bugge TH. Potent antitumor activity of a urokinase-activated engineered anthrax toxin. Proc Natl Acad Sci USA 2003; 100 657-662.

25. Phillips DD, Fattah RJ, Crown D, Zhang Y, Liu S, Moayeri M et al. Engineering anthrax toxin variants that exclusively form octamers and their application to targeting tumors. J Bio Chem 2013; 288: 9058-9065.

26. Liu S, Redeye V, Kuremsky JG, Kuhnen M, Molinolo A, Bugge TH et al. Intermolecular complementation achieves high-specificity tumor targeting by anthrax toxin. Nat Biotechnol 2005; 23: 725-730.

27. DiRienzo JM, Cao L, Volgina A, Bandelac G, Korostoff J. Functional and structural characterization of chimeras of a bacterial genotoxin and human type I DNAse. FEMS Microbiol Lett 2009; 291: 222-231.
28. Shenker BJ, Dlakic M, Walker LP, Besack D, Jaffe E, LaBelle E et al. A novel mode of action for a microbial-derived immunotoxin: the cytolethal distending toxin subunit $B$ exhibits phosphatidylinositol 3,4,5-triphosphate phosphatase activity. J Immunol 2007; 178: 5099-5108.

29. Damek-Poprawa M, Volgina A, Korostoff J, Sollecito TP, Brose MS, O’Malley BW Jr. et al. Targeted inhibition of CD133 + cells in oral cancer cell lines. J Dental Res 2011; 90: 638-645.

30. Shenker BJ, Boesze-Battaglia K, Zekavat A, Walker L, Besack D, Ali H. Inhibition of mast cell degranulation by a chimeric toxin containing a novel phosphatidylinositol-3,4,5triphosphate phosphatase. Mol Immunol 2010; 48: 203-210.

31. Damek-Poprawa M, Jang JY, Volgina A, Korostoff J, DiRienzo JM. Localization of Aggregatibacter actinomycetemcomitans cytolethal distending toxin subunits during intoxication of live cells. Infect Immun 2012; 80: 2761-2770.

32. Hu X, Nesic D, Stebbins CE. Comparative structure-function analysis of cytolethal distending toxins. Proteins 2006; 62: 421-434.

33. Nishikubo S, Ohara M, Ueno Y, Ikura M, Kurihara H, Komatsuzawa $\mathrm{H}$ et al. An N-terminal segment of the active component of the bacterial genotoxin cytolethal distending toxin $B$ (CDTB) directs CDTB into the nucleus. J Biol Chem 2003; 278: 50671-50681.

34. Fuchs H, Bachran C, Li T, Heisler I, Durkop H, Sutherland M. A cleavable molecular adapter reduces side effects and concomitantly enhances efficacy in tumor treatment by targeted toxins in mice. J Control Release 2007; 117: 342-350.

35. Liu S, Leung HJ, Leppla SH. Characterization of the interaction between anthrax toxin and its cellular receptors. Cell Microbiol 2007; 9: 977-987.

36. Weinstein JN, Myers TG, O'Connor PM, Friend SH, Fornace AJ Jr, Kohn KW et al. An information-intensive approach to the molecular pharmacology of cancer. Science 1997: 275: 343-349.

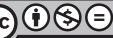

Cell Death and Disease is an open-access journal published by Nature Publishing Group. This work is licensed under a Creative Commons Attribution-NonCommercialNoDerivs 3.0 Unported License. To view a copy of this license, visit http://creativecommons.org/licenses/by-nc-nd/3.0/

Supplementary Information accompanies this paper on Cell Death and Disease website (http://www.nature.com/cddis) 